Anuario Latinoamericano Ciencias Políticas

y Relaciones Internacionales vol. 8, 2019

pp. 341-343

DOI: $10.17951 / \mathrm{al} .2019 .8 .341-343$

\section{La VI edición del Simposio Internacional "Encuentros Científicos con América Latina" en la Facultad de Ciencias Políticas y Periodismo de la Universidad Maria Curie-Skłodowska, 22-25 de octubre 2019, Lublin, Polonia}

\section{The 6th Edition of the International Symposium "Scientific Meetings with Latin America" at the Faculty of Political Science and Journalism of Maria Curie-Skłodowska University, October 22-25, 2019, Lublin, Poland}

Los "Encuentros Científicos con América Latina" es un evento cíclico organizado en la Facultad de Ciencias Políticas y Periodismo de la Universidad Maria CurieSkłodowska desde el año 2015. La idea de organizar los simposios internacionales en la UMCS fue concebida por la Prof. Dra. hab. Katarzyna Krzywicka, la directora y editora en jefe de la revista científica Anuario Latinoamericano - Ciencias Políticas y Relaciones Internacionales. Su objetivo es divulgar el conocimiento sobre la región de América Latina entre los científicos y estudiantes interesados en diferentes áreas de las ciencias políticas y también darles la oportunidad de participar en un debate con los representantes del mundo académico de diferentes países.

La sexta edición de los "Encuentros Científicos con América Latina” tuvo lugar los días 22-25 de octubre 2019 en Lublin, Polonia. El tema principal fue "Políticas de seguridad de los Estados de América Latina". En el simposio participaron, aparte de los representantes de la Facultad de Ciencias Políticas y Periodismo de la UMCS, los investigadores de otras universidades polacas de Łódź, Przemyśl y Cracovia, y también los científicos de los centros académicos extranjeros de Gran Bretaña y Lituania.

El simposio fue inaugurado por el Prof. Dr. hab. Marek Pietraś, el director del Instituto de Ciencias Políticas y Administración, de la Facultad de Ciencias Políticas y Periodismo de la Universidad Maria Curie-Skłodowska. La introducción al tema del simposio fue llevada a cabo por la Prof. Dra. hab. Katarzyna Krzywicka, quien fue también encargada de moderar todas las sesiones. Además, la Prof. K. Krzywicka realizó la presentación de la revista Anuario Latinoamericano - Ciencias Políticas y Relaciones Internacionales, de la cual fue fundadora en 2014 y desde el principio ha desempeñado la función de directora y editora en jefe. Anuario Latinoamericano es la primera publicación periódica con el perfil politológico en el área de estudios latinoamericanos 
editada en Polonia y ha conseguido 20 puntos en la lista de revistas científicas polacas reconocidas por su impacto en la divulgación científica y apoyadas por el Ministerio de Ciencia y Educación Superior de la República de Polonia. La Prof. Krzywicka presentó el alcance temático y las cuestiones relacionadas con la evaluación y divulgación de la revista.

El primer día del simposio tuvieron lugar dos conferencias. La primera fue impartida por el Dr. Michał Stelmach del Departamento de Estudios Latinoamericanos y Comparativos, la Facultad de Estudios Internacionales y Ciencias Políticas de la Universidad de Łódź, Polonia. El tema de su conferencia fue "Entre la militarización y privatización. Modelos de la política de seguridad pública en los países de América Latina en el siglo XXI". El Dr. Stelmach presentó las tendencias dominantes en el sector de la seguridad pública, enfocándose en los problemas relacionados con el alto índice de homicidios en América Latina, poca eficacia y baja confianza en la policía, que está cada vez más militarizada, así como el funcionamiento de las agencias de seguridad privadas.

La segunda conferencia, titulada "La política de seguridad de Brasil en el siglo XXI", fue impartida por la Dra. Edyta Chwiej del Instituto de Ciencias Políticas y Relaciones Internacionales de la Universidad Jaguelónica, Cracovia, Polonia. La autora empezó por presentar los factores que tienen impacto en la actual política de seguridad brasileña, luego pasó al análisis de la Estrategia Nacional de Defensa, y terminó indicando las prioridades de la administración del presidente Jair Bolsonaro.

El día siguiente de los "Encuentros Científicos con América Latina” tuvieron lugar tres conferencias presentadas por los representantes del Departamento de Relaciones Internacionales, el Instituto de Ciencias Políticas y Administración, de la Facultad de Ciencias Políticas y Periodismo de la Universidad Maria CurieSkłodowska. La primera ponencia, impartida por la Mag. Maria Ochab, fue dedicada a la cuestión de la cooperación entre los países de América Latina con la Unión Europea en el área de la seguridad humana. La conferencista presentó varias iniciativas emprendidas conjuntamente por los países latinoamericanos y diferentes entidades institucionales de la Unión Europea, indicando los medios, alcance temático y bases legales para esta cooperación. La segunda ponencia, titulada "The Kingpin Strategy como un ejemplo del combate contra el crimen organizado", impartida por la Mag. Aleksandra Jargiełło explicó en que consiste, como fue implementada y que resultados logró la estrategia de "descabezamiento" de grupos criminales, promovida por las administraciones de Felipe Calderón y Enrique Peña Nieto en México. La tercera ponencia del representante de la UMCS fue titulada "La política de la seguridad energética de Brasil”. El Mag. Michał Dziaduch al principio presentó el panorama del sector energético mundial para pasar a la actual situación en cuanto a los recursos energéticos de los que dispone Brasil y las estrategias estatales adoptadas para proporcionar la seguridad energética del país.

Durante el tercer día del simposio fueron impartidas tres ponencias por los conferenciantes invitados. El Dr. hab. Karol Derwich, del Departamento de Estudios Latinoamericanos de la Universidad Jaguelónica, Cracovia, Polonia, 
analizó las estrategias de la seguridad pública en México en el siglo XXI desde el punto de vista de sus objetivos y resultados obtenidos. La primera parte de su ponencia fue dedicada al problema de narcotráfico. Luego sometió al análisis las estrategias implementadas por el presidente Vicente Fox (2000-2006), Felipe Calderón (2006-2012), y Enrique Peña Nieto (2012-2016), para terminar con la presentación de la situación actual de la seguridad pública bajo el gobierno de Andrés Manuel López Obrador. La segunda conferencia fue impartida por la Mag. Ieva Giedraitytė del Instituto de Relaciones Internacionales y Ciencias Políticas de la Universidad de Vilna, Lituania. Su ponencia fue titulada "From the migration crisis to the migration crisis: five years of the US Strategy for Engagement in Central America. Has it failed?". La conferenciante invitada abordó el tema de la relación entre las crisis migratorias en América Central y la así llamada Estrategia de Relacionamiento de Estados Unidos para Centroamérica, adoptada por la administración de los Estados Unidos para proteger sus intereses y promover la seguridad y estabilidad en la región. La última conferencia fue dedicada al tema del desarrollo del crimen organizado transnacional en la región de la Triple Frontera (Argentina, Brasil y Paraguay). El Dr. Paweł Trefler del Instituto de Relaciones Internacionales y ciencias Políticas, de la Escuela Superior Estatal de Europa Oriental de Przemyśl, Polonia, llevó a cabo un análisis de las raíces del problema, así como el impacto causado por el crimen organizado en la mencionada zona fronteriza.

Los "Encuentros Científicos con América Latina” terminaron con la conferencia del Dr. Carlos A. Pérez Ricart, del Latin American Center, St. Antony's College, Universidad de Oxford, quien ha disertado sobre el tema "Understanding Mexico`s Drug Violence: Historical and Contemporary Perspectives”. El ponente partió del panorama histórico, presentando como se iba desarrollando la guerra contra el narcotráfico para aproximarse a la explicación plausible de la crisis de extrema violencia en México. El Dr. Pérez Ricart indicó también el papel de México en el tráfico global de drogas y el rol de los Estados Unidos en la crisis actual.

La sexta edición del simposio internacional "Encuentros Científicos con América Latina”, organizada en la Universidad Maria Curie-Skłodowska, proporcionó a los participantes una buena oportunidad para compartir los resultados de sus investigaciones, intercambiar las ideas y pensar sobre los futuros proyectos y posible colaboración. Como siempre, la Prof. Katarzyna Krzywicka animó a los participantes a pensar en la publicación de artículos en Anuario Latinoamericano e invitó a todos para los futuros eventos organizados por el Equipo Editorial de la revista y la Facultad de Ciencias Políticas y Periodismo de la UMCS en Lublin.

Maria Ochab

Facultad de Ciencias Políticas y Periodismo

Universidad Maria Curie-Skłodowska

Lublin, Polonia

$\triangle$ maria.ochab@umcs.edu.pl

https://orcid.org/0000-0002-0061-3558

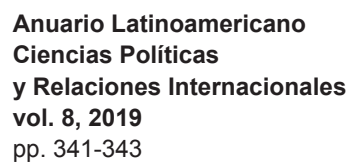

DOI: 10.17951/al.2019.8.341-343 